\title{
An Integrative Network Biology Approach to Evaluate the Role of Endoplasmic Reticulum Stress Response in Obese Type 2 Diabetes
}

\author{
Anup Mammen Oommen, Usha Narayanan, and M. R. Jagannath \\ Systems Biology Group, Connexios Life Sciences Private Limited, No.49 First Main Road, 3rd Phase, JP Nagar, \\ Bangalore 560078, India \\ Correspondence should be addressed to M. R. Jagannath, m.r.jagannath@connexios.com
}

Received 1 February 2012; Accepted 15 March 2012

Academic Editors: A. Chiarini and A. Colanzi

Copyright ( 2012 Anup Mammen Oommen et al. This is an open access article distributed under the Creative Commons Attribution License, which permits unrestricted use, distribution, and reproduction in any medium, provided the original work is properly cited.

\begin{abstract}
Extracellular/intracellular stimuli can influence eukaryotic cell function through organelles that regulate critical signaling pathways. The endoplasmic reticulum (ER), for example, impacts cellular processes including protein synthesis, folding and secretion; amino acid transport; apoptosis; cell proliferation; lipid synthesis across major cell types in response to stimuli such as accumulation of misfolded proteins and glucose deprivation. Dysregulated signaling pathways underlying the ER-mediated processes mentioned above have been linked to disease conditions such as diabetes, obesity, and Alzheimer's disease. Our current understanding, however, lacks a detailed network view that integrates organelle-mediated pathway dysregulation with cellular processes and disease pathogenesis. In this report, we introduce an integrative network biology approach that combines ER-stress response pathways with basic cellular processes using data from peer-reviewed literature. As an example, we apply our systems biology approach to study the role of ER stress in pancreatic $\beta$ cells under obese diabetic conditions, generate testable hypotheses, and provide novel insights into $\beta$-cell pathogenesis.
\end{abstract}

\section{Introduction}

The endoplasmic reticulum (ER) is a $3 \mathrm{D}$ network of tubules and cisternae divided into the nuclear envelope, rough ER, and smooth ER, each with a distinct function. The ER performs numerous cellular functions including synthesis of secreted and membrane proteins, biosynthesis of phospholipids, cholesterol, and steroids, and degradation of glycogen and calcium homeostasis. Factors such as oxidative stress, ischemia, and increased load of nascent or misfolded proteins or perturbations in calcium homeostasis can interfere with normal ER function leading to an accumulation of misfolded proteins [1]. This process is called "ER stress" and activates the unfolding protein response (UPR), which (a) helps restore normal cellular function by stopping protein translation, and (b) activates signaling pathways to increase production of molecular chaperones involved in protein folding [2]. If the disruption is prolonged, the UPR tries to turn on the apoptotic pathway [2]. Thus, the UPR safeguards protein synthesis, posttranslational modifications, folding and secretion, calcium storage and signaling, and lipid biosynthesis. Stresses that trigger UPR include elevated secretory protein synthesis; overexpression and/or accumulation of mutant proteins [3]; aberrant $\mathrm{Ca}^{2+}$ regulation [4]; hypoxia [5]; altered glycosylation [6]; ischemia [7]; viral infections [8]; redox state of ER compartment [9]; overloading of cholesterol [10]; glucose deprivation [11].

The ER is a key organelle in pancreatic $\beta$ cells and influences insulin synthesis, reduces oxidative stress, and preserves $\beta$-cell differentiation $[12,13]$. Studies in the pancreatic $\beta$-cell line, MIN6 have demonstrated that under inflammatory conditions, nitric-oxide-mediated calcium dysregulation induces ER stress and subsequent $\beta$-cell apoptosis [14]. The T2DM mouse model, Akita which spontaneously develops hyperglycemia with reduced $\beta$-cell mass shows accumulation of a mutation-induced, misfolded pro-insulin, and subsequent ER stress leading to reduced $\beta$-cell mass [15]. ER stress has been linked to glucolipotoxicity and inflammatory signal-mediated beta cell apoptosis through islet studies from T2DM patients and rodents; INS1 cell lines 
and the pancreatic cell line, BRIN-BDII link [16-19]. These data indicate that the ER is a highly sensitive organelle that modulates $\beta$ cell function by regulating apoptotic pathways in response to physiological and pathological conditions.

The pancreatic islet $\beta$ cells are highly responsive to glucose-mediated insulin secretion (GSIS), which is required for plasma glucose homeostasis. Recent data from human clinical trials and experimental studies in animal models show that under conditions of metabolic and systemic stress, loss of $\beta$-cell function reduces GSIS resulting in a subsequent loss of islet mass and accelerating the transition from hyperglycemia to T2DM $[20,21]$.

A growing area of research in type 2 diabetes is the use of in silico models to better understand disease pathogenesis and predict novel targets of therapeutic value. These in silico models (comparable to a Basic Input Output System) generate a complex pattern of cellular outcomes integrated across tissue and organismal levels by processing information and communicating it to other interconnected modules. A major challenge in this field of research is to understand the physiological relevance of cellular outcomes and address feedback loops formed by interactions within/between components of cellular subsystems. This challenge is made even greater by the physiological conditions.

In this paper, we use an integrative network biology approach to study the role of the ER protein folding machinery and UPR in regulating $\beta$-cell apoptosis in the obese diabetic model, BTBR. We introduce our proprietary knowledgebase for ER stress signaling, which has been built by integrating ER stress-specific experimental data across cell types and species from peer-reviewed literature. We validate the network by discussing current hypotheses in obesityassociated type 2 diabetes and provide novel hypotheses that can be tested to gain a better understanding of disease pathogenesis.

\section{Methods: Details of the Connexios Network Biology Platform}

The Connexios Network Biology Platform is an integrated repository of molecular events spanning numerous cellular pathways across core metabolic tissues including adipose, liver, and muscle. It has a modular design where each module represents the molecular network of a cellular function and is generated by integrating molecular interactions extracted from peer-reviewed literature. As seen in Figure 1, we developed the modules through manual curation of experimental data on signaling pathways; transcriptional and posttranscriptional regulatory pathways and metabolic pathways. These modules were then integrated across cellular and organ-level physiologies to generate in silico cellular models. We currently have $>300$ modules that can be used in various combinations to generate specific cellular/tissue networks. Broadly, the cell types covered by Connexios Network Biology Platform include adipocytes, hepatocytes, myotubes, pancreatic islet $\beta$ cells, endothelial cells, and macrophages. In these cell types, we have generated network models of disease states by analyzing the effects of perturbations on cellular networks using gene expression data from tissue samples of rodent models and human subjects (Figure 1). The gene expression data in the Connexios Array Database have been obtained from public sources including GEO, DGAP, and EPConDB.

\subsection{Applying the Connexios Integrative Network Biology} Approach to Study ER Stress in Disease Biology. The Connexios ER stress module (Figure 2; details in the supplementary section) includes:

(a) components of the ER machinery involved in protein import, folding, and maturation;

(b) multiple physiological inputs across experimental systems and mutations that induce accumulation of misfolded proteins in the ER;

(c) the UPR arm along with details of the transcriptional and posttranslational regulation and feedback mechanisms that regulate misfolded protein accumulation, stress, and other cellular processes;

(d) ER stress-induced programmed cell death.

When the ER protein folding, processing, and maturation machinery fail to cope with the increasing load of nascent or misfolded proteins, the ER experiences "stress". If unchecked, it results in the accumulation of misfolded proteins initiating an unfolded protein response (UPR). The three arms of UPR include: dimerisation and activation of pancreatic eIF $2 \alpha$ kinase (PEK) or PKR-like eIF2 $\alpha$ A-PERK (eIF2AK3); dimerisation and activation of inositol response element 1 (IRE1); release/processing of ATF6 [22-24]. The UPR is an internal sensor of the quantity and quality of the ER protein and mediates feedback regulation to the ER. The immediate impact of the UPR-mediated responses mentioned above are regulation of protein load through a PERKeIF2-mediated inhibition of protein synthesis; IRE1-P58IPKmediated mRNA degradation [25] a cytosolic, proteasomal degradation of nascent polypeptides [26]. Coordinated regulation of UPR response genes by the ATF4 (activated by PERK), ATF6, and XBP1 (activated by IRE1) transcriptional factors may increase expression levels of chaperones and cochaperones to ensure proper protein folding and trigger degradation machinery to clear misfolded proteins. Harding et al. showed that the ATF4 and PERK arms of UPR protect cells against oxidative stress by increasing aminoacid supply for protein synthesis and glutathione biosynthesis [27]. The PERK arm of UPR activates NRF2, a major transcription factor for antioxidant genes that protects cells from stressinduced apoptosis [28-30]. Similarly, Bommiasamy et al. [31] and Sriburi et al. [32] have identified a role for the ATF6 and XBP1 transcription factors in regulating ER membrane biogenesis by increasing phosphatidylcholine biosynthesis [33]. Genes induced in response to multiple UPR arms have been listed in Table S1 in Supplementary Material available online at doi:10.5402/2012/278636.

Prolonged ER stress triggers PERK-, ATF6- and IRE1mediated proapoptotic events. Two major signaling cascades activated by the UPR to initiate apoptosis are (a) ATF4/6 signaling pathways activating DDIT3 (growth-arrest and 


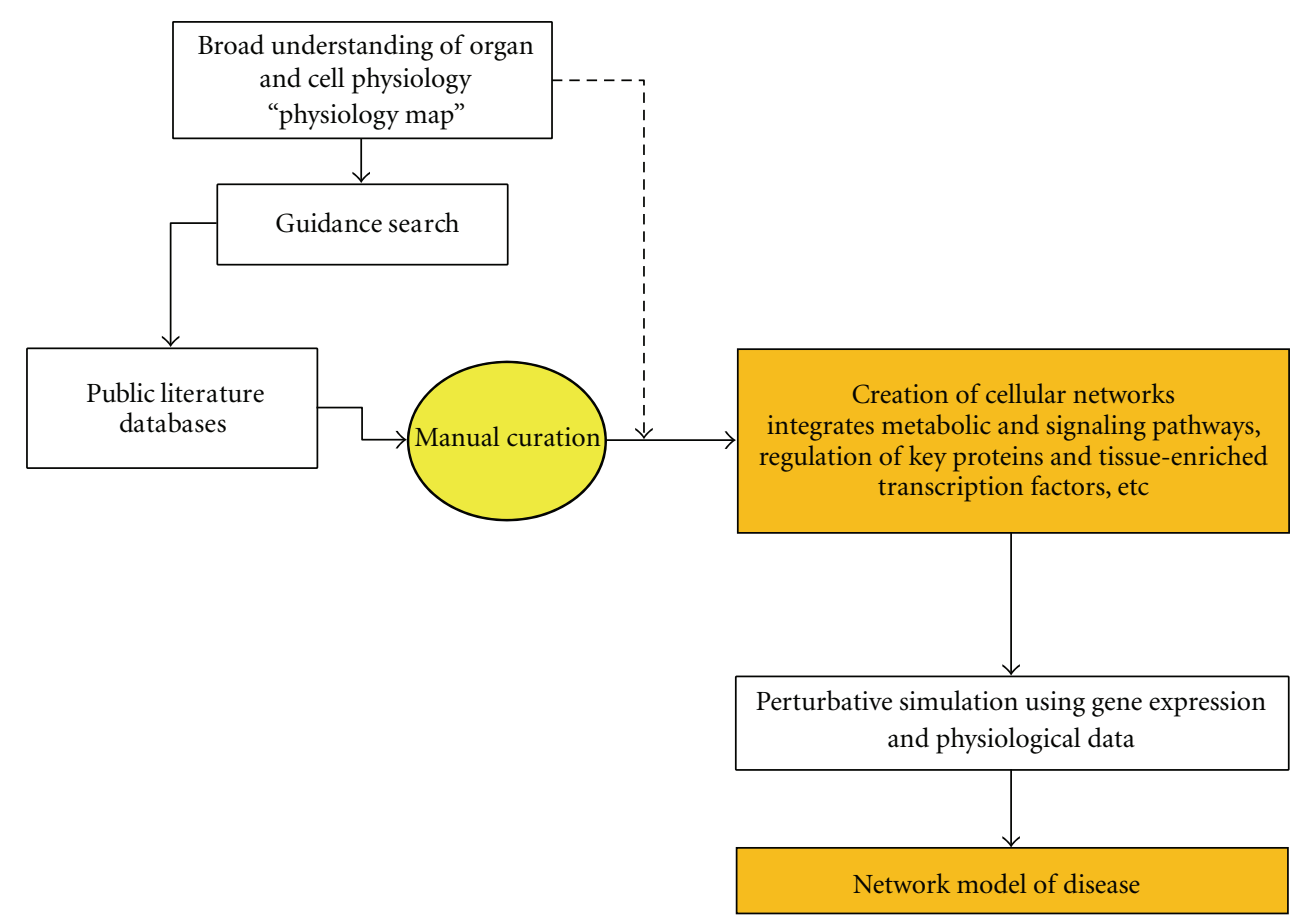

FIGURE 1: Flow chart showing the process flow used to generate a disease model using an integrative network biology approach. Experimental data from peer-reviewed literature associated with metabolic and cell signalling networks were systematically integrated to generate in silico cellular models. These molecular networks could be mapped to disease biology using network abstraction.

DNA damage inducible gene 153) and (b) the JNK pathway (reviewed in [34]). DDIT3 reduces expression of BCL2, an antiapoptotic gene [35]. Similarly, the p38/MAPK and JNK cascades, known mediators of stress-induced apoptosis, are triggered by IRE1 in response to ER stress [36]. Activation of p38/MAPK also potentiates DDIT3 transcriptional activity by phosphorylation [37].

Systematic integration of the above data on ER protein import, folding, maturation, degradation, and the UPR arm provides a better understanding of ER stress signaling and forms the basis of the Connexios ER stress network. As such, it offers novel insights into regulation of cell function under normal and pathophysiological conditions. In this report, we use the Connexios ER stress network to study gene expression data from pancreatic islets of the obese diabetic BTBR mouse model (GEO dataset: GSE 10785) [39] and develop network predictions to help identify novel testable hypotheses.

\section{Results and Discussion}

To examine UPR machineries and their impact on beta cell pathology, we used the Connexios ER stress network module and analyzed genomic data from pancreatic $\beta$ cells of obese diabetic BTBR mice (GSE 10785-GEO datasets). We used the Micro_stats_package to compute the $m$ value and $P$ value as described in supplementary information. Key genes that showed statistically significant changes in expression $(m \geq$ $0.3, P=0.05)$ are listed in Table 1 .

The BTBR model, rendered genetically obese $(\mathrm{ob} / \mathrm{ob})$, develops severe type 2 diabetes on a high fat diet $[40,41]$.
Impaired $\beta$-cell function characterized by a failure to maintain a sufficient number of glucose-sensitive $\beta$ cells is an essential component of diabetes [38]. The four phases of $\beta$-cell deterioration include: (1) successful adaption to increased demand, (2) mild decompensation, (3) severe decompensation, and (4) decompensation with damage [42].

Clee et al. [38] reported that nearly half the pancreatic $\beta$ cells are decompensated in 10-week-old BTBR male mice (Figure 3). Briefly, they found that in the BTBR mouse model, plasma glucose levels increased with age whereas the plasma insulin levels decreased with age. The difference between plasma glucose and insulin levels over control B6 and BTBR mice were most pronounced at 10 weeks, with effects being more prominent in males as opposed to females. Taking these data in conjunction with the beta cell deterioration process predicted by Weir and Bonnerweir [42], we hypothesized that $\beta$ cells in BTBR mice transit through the above four stages of deterioration.

To understand $\beta$-cell deterioration in obesity-induced type 2 diabetic BTBR mice, we studied microarray data from BTBR mice and found increased expression of UPR genes, PERK and IRE1, and reduced expression of the DDIT3 and ATF4 transcription factors. Reduction in ATF4 correlated with a reduction in amino-acid transport as well as reduced expression of metabolic genes [43], namely, arginine, glutamate transporters (SLCIA4 and SLC3A2) and genes involved in asparagine and $\mathrm{N}$-methyl glycine synthesis. There was a similar reduction in YARS, the tRNA charging enzyme [43] involved in translation. We also found an upregulation of the $26 \mathrm{~S}$ proteasomal regulatory subunit, Derlin, and the mannosidase family of ER degradation 


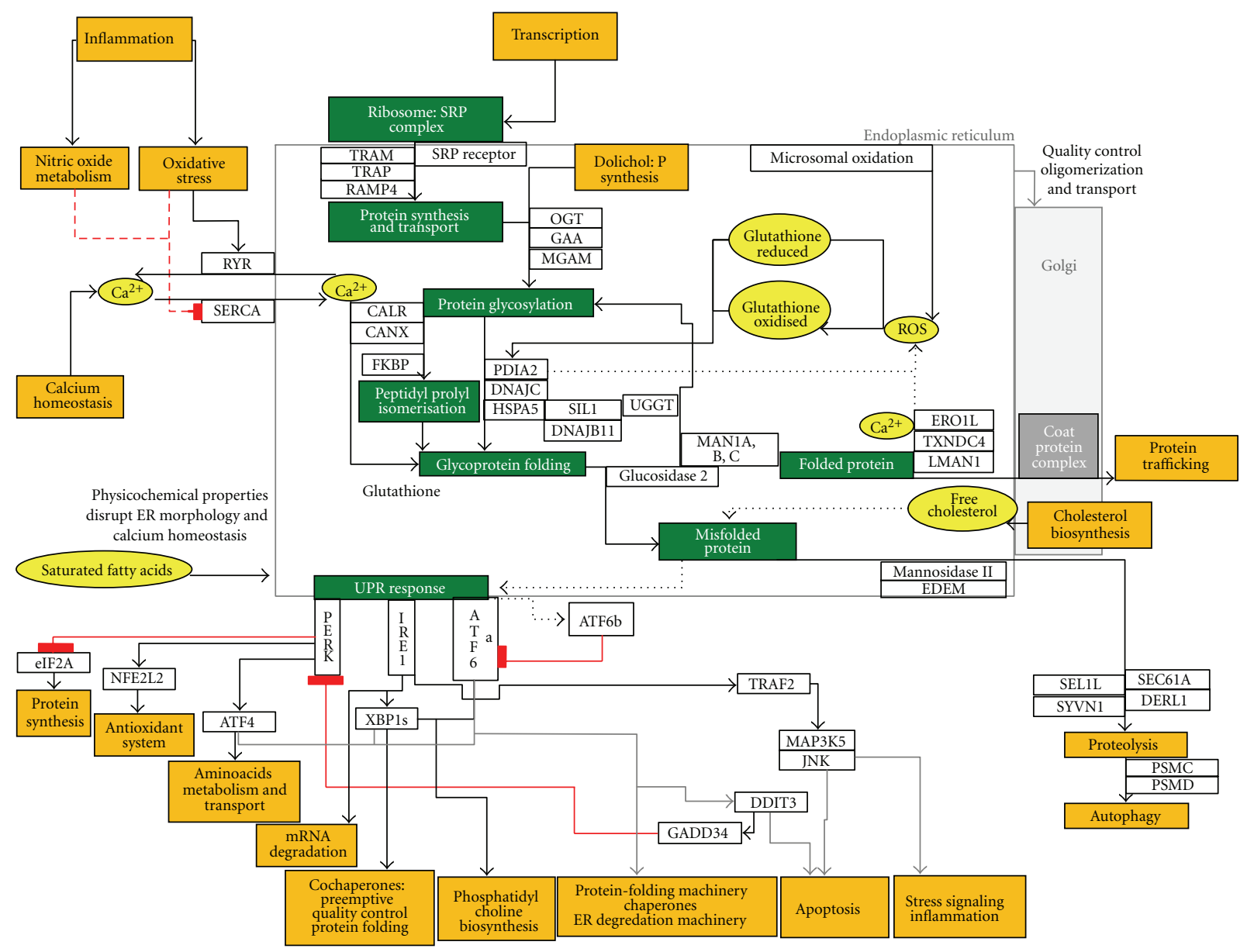

FIgURE 2: Overview of the ER machinery and its link to major cellular processes from the Connexios Network Knowledge Database. Experimental data on ER stress were systematically curated and integrated with multiple cellular processes that have been developed as individual modules on the Connexios Network Biology platform. This generates an integrated view of ER stress signaling presented as the Connexios ER stress module. In this figure, orange boxes represent cellular processes linked to ER stress; green boxes indicate glycoprotein folding and UPR machinery; solid black/grey arrows show direct activation; dotted bar-headed red lines show indirect activation; solid barheaded red lines indicate direct inhibition; the central black rectangle represents the endoplasmic reticulum; the grey shaded box represents the Golgi and the yellow ovals represent secondary molecular inputs required for protein folding.

machineries [44]. Since these proteins are known to degrade misfolded glycoproteins, it suggests that an accumulation of misfolded proteins in the ER in the obese diabetic mouse model.

We also found that the DNAJ family of cochaperones (DNAJB11, DNAJC10, and DNAJC3) [45]; HSPA5; FK506binding proteins (FKBP 2, 9, 10) [46]; the protein disulfide isomerases [47] were upregulated. These proteins are known to ensure proper folding inside the ER lumen further suggesting that an increased load of misfolded proteins in the ER in the obese diabetic mouse model.

The thioredoxin domain-containing proteins (TXNDC5/ 12) [48] were significantly increased suggesting reduced ROS generation in the ER in the BTBR mouse model. We also found a significant upregulation of the glycoprotein quality control genes known to be critical for glycoprotein turnover during folding and degradation.
Genes involved in dolichol phosphate mannose synthesis and transfer reactions (Table 1) were also upregulated. Interestingly, the phosphatidylcholine biosynthesis gene (PCYTIA) induced by the UPR and crucial for ER membrane biogenesis [49] was downregulated implying that the ER's capacity to accommodate the increased overload of misfolded proteins is compromised in the BTBR model.

We also detected increased levels of quality control genes, which trim mannosidase linkages on complex glycoproteins in the BTBR mice. These genes are implicated in glycoprotein turnover during ER stress [44]. Relevance of this finding for $\beta$-cell glycoprotein turnover needs to be further examined.

In summary, the 10-week-old BTBR mice showed an increase in ER degradation machinery, FK506 binding proteins, protein disulfide isomerases, and thioredoxin domaincontaining proteins in response to ER stress. These proteins are involved in regulating misfolded protein accumulation 
TABle 1: Partial gene expression data of UPR responsive genes in $\beta$ cells of obese diabetic model. Gene expression data obtained from pancreatic $\beta$ cells of obese BTBR diabetic model (GSE 10785) obtained from GEO datasets; $P$ value $=0.05$. The $m$ value, $P$ value were computed using Micro_stats_package. Negative and positive values represent genes which are downregulated and upregulated, respectively, when compared to control animals.

\begin{tabular}{|c|c|c|}
\hline Gene symbol & $m$ value & Gene name \\
\hline \multicolumn{3}{|r|}{ UPR Arm } \\
\hline DDIT3 & -1.628 & DNA-damage-inducible transcript 3 \\
\hline ERN1 & 1.067 & Endoplasmic reticulum to nucleus signaling 1 \\
\hline ERN2 & 0.405 & Endoplasmic reticulum to nucleus signaling 2 \\
\hline EIF2AK3 & 0.713 & Eukaryotic translation initiation factor 2-alpha kinase 3 \\
\hline ATF4 & -0.577 & Activating transcription factor 4 (tax-responsive enhancer element B67) \\
\hline \multicolumn{3}{|r|}{ Amino acid transport and metabolism } \\
\hline SLC1A4 & -1.575 & Solute carrier family 1 (glutamate/neutral amino acid transporter), member 4 \\
\hline SLC3A2 & -0.726 & Solute carrier family 3 (activators of dibasic and neutral amino acid transport), member 2 \\
\hline ASNS & -0.757 & Asparagine synthetase (glutamine-hydrolyzing) \\
\hline GNMT & -1.243 & Glycine N-methyltransferase \\
\hline YARS & -0.655 & Tyrosyl-tRNA synthetase \\
\hline \multicolumn{3}{|r|}{ Proteosomal machinery } \\
\hline PSMD13 & 0.416 & Proteasome (prosome, macropain) 26S subunit, non-ATPase, 13 \\
\hline PSMD3 & 0.424 & Proteasome (prosome, macropain) 26S subunit, non-ATPase, 3 \\
\hline PSMD2 & 0.436 & Proteasome (prosome, macropain) 26S subunit, non-ATPase, 2 \\
\hline PSMD8 & 0.46 & Proteasome (prosome, macropain) 26S subunit, non-ATPase, 8 \\
\hline PSMC1 & 0.498 & Proteasome (prosome, macropain) 26S subunit, ATPase, 1 \\
\hline PSMD14 & 0.507 & Proteasome (prosome, macropain) 26S subunit, non-ATPase, 14 \\
\hline PSMC4 & 0.514 & Proteasome (prosome, macropain) 26S subunit, ATPase, 4 \\
\hline PSMD7 & 0.308 & Proteasome (prosome, macropain) 26S subunit, non-ATPase, 7 \\
\hline PSMC3 & 0.312 & Proteasome (prosome, macropain) 26S subunit, ATPase, 3 \\
\hline PSMC2 & 0.414 & Proteasome (prosome, macropain) 26S subunit, ATPase, 2 \\
\hline PSMD4 & 0.341 & Proteasome (prosome, macropain) 26S subunit, non-ATPase, 4 \\
\hline \multicolumn{3}{|r|}{ ER degradation machinery } \\
\hline EDEM2 & 1.859 & ER degradation enhancer, mannosidase alpha-like 2 \\
\hline EDEM3 & 0.713 & ER degradation enhancer, mannosidase alpha-like 3 \\
\hline DERL1 & 0.322 & Der1-like domain family, member 1 \\
\hline DERL2 & 0.588 & Der1-like domain family, member 2 \\
\hline DERL3 & 1.102 & Der1-like domain family, member 3 \\
\hline SYVN1 & 0.487 & Synovial apoptosis inhibitor 1 , synoviolin \\
\hline \multicolumn{3}{|r|}{ Quality control and protein folding } \\
\hline DNAJB9 & 0.501 & DnaJ (Hsp40) homolog, subfamily B, member 9 \\
\hline DNAJB11 & 0.871 & DnaJ (Hsp40) homolog, subfamily B, member 11 \\
\hline DNAJC10 & 0.972 & DnaJ (Hsp40) homolog, subfamily C, member 10 \\
\hline DNAJC3 & 1.564 & DnaJ (Hsp40) homolog, subfamily C, member 3 \\
\hline HSPA5 & 0.698 & Heat shock $70 \mathrm{kDa}$ protein 5 (glucose-regulated protein, $78 \mathrm{kDa}$ ) \\
\hline ERP29 & 1.886 & Endoplasmic reticulum protein 29 \\
\hline FKBP10 & 0.47 & FK506-binding protein $10,65 \mathrm{kDa}$ \\
\hline FKBP2 & 0.868 & FK506-binding protein 2, $13 \mathrm{kDa}$ \\
\hline FKBP9 & 0.741 & FK506-binding protein $9,63 \mathrm{kDa}$ \\
\hline PDIA3 & 0.58 & Protein disulfide isomerase family A, member 3 \\
\hline PDIA4 & 1.365 & Protein disulfide isomerase family A, member 4 \\
\hline PDIA5 & 0.842 & Protein disulfide isomerase family A, member 5 \\
\hline PDIA6 & 0.416 & Protein disulfide isomerase family A, member 6 \\
\hline PPIB & 0.619 & Peptidylprolyl isomerase B (cyclophilin B) \\
\hline
\end{tabular}


Table 1: Continued.

\begin{tabular}{|c|c|c|}
\hline Gene symbol & $m$ value & Gene name \\
\hline SIL1 & 1.383 & SIL1 homolog, endoplasmic reticulum chaperone (S. cerevisiae) \\
\hline TXNDC12 & 0.614 & Thioredoxin domain containing 12 (endoplasmic reticulum) \\
\hline TXNDC5 & 0.779 & Thioredoxin domain containing 5 (endoplasmic reticulum) \\
\hline LMAN1 & 0.55 & Lectin, mannose binding, 1 \\
\hline MAN1B1 & 0.481 & Mannosidase, alpha, class $1 \mathrm{~B}$, member 1 \\
\hline MAN2A1 & 1.277 & Mannosidase, alpha, class 2A, member 1 \\
\hline MAN2B1 & 0.318 & Mannosidase, alpha, class 2B, member 1 \\
\hline MAN2B2 & 0.576 & Mannosidase, alpha, class 2B, member 2 \\
\hline MGAM & -1.497 & Maltase-glucoamylase (alpha-glucosidase) \\
\hline UGGT1 & 0.683 & UDP-glucose glycoprotein glucosyltransferase 1 \\
\hline UGGT2 & 0.395 & UDP-glucose glycoprotein glucosyltransferase 2 \\
\hline PDIA2 & -1.207 & protein disulfide isomerase family A, member 2 \\
\hline ERO1LB & -1.902 & ERO1-like $\beta$ (S. cerevisiae) \\
\hline \multicolumn{3}{|r|}{ ER transport } \\
\hline TMED4 & 0.787 & Transmembrane emp24 protein transport domain containing 4 \\
\hline TRAM1 & 0.41 & Translocation associated membrane protein 1 \\
\hline ERGIC1 & 0.97 & Endoplasmic reticulum-Golgi intermediate compartment (ERGIC) 1 \\
\hline \multicolumn{3}{|r|}{ Phosphatidylcholine biosynthesis } \\
\hline PCYT1A & -0.542 & Phosphate cytidylyltransferase 1 , choline, alpha \\
\hline \multicolumn{3}{|r|}{ Dolichol-phosphate-mannose synthesis and transfer reactions } \\
\hline ALG12 & 0.324 & Asparagine-linked glycosylation 12, alpha-1,6-mannosyltransferase homolog (S. cerevisiae) \\
\hline DHDDS & 0.382 & Dehydrodolichyl diphosphate synthase \\
\hline DPAGT1 & 0.756 & $\begin{array}{l}\text { Dolichyl-phosphate (UDP-N-acetylglucosamine) N-acetylglucosaminephosphotransferase } 1 \\
\text { (GlcNAc-1-P transferase) }\end{array}$ \\
\hline PMM2 & 0.544 & Phosphomannomutase 2 \\
\hline PMM1 & 0.701 & Phosphomannomutase 1 \\
\hline FDPS & 0.982 & Farnesyl diphosphate synthase \\
\hline ALG11 & 0.378 & Asparagine-linked glycosylation 11, alpha-1,2-mannosyltransferase homolog (yeast) \\
\hline ALG14 & 0.713 & Asparagine-linked glycosylation 14 homolog (S. cerevisiae) \\
\hline MPDU1 & 0.47 & Mannose-P-dolichol utilization defect 1 \\
\hline DPM3 & 0.884 & Dolichyl-phosphate-mannosyltransferase polypeptide 3 \\
\hline
\end{tabular}

in a feedback mechanism to UPR [2]. Gene expression data from the obese diabetic BTBR mice also showed a significant increase in the expression of the PERK and IRE1 arms of UPR compared to the lean, littermate controls. Together, these findings suggest that accumulation of misfolded proteins in the ER is increased in the BTBR obese diabetic mice indicative of ER stress. This is the first report providing a robust correlation between ER stress and beta cell dysfunction under obese diabetic conditions using the BTBR mice. Previously, Karaskov et al. and Marchetti et al. showed that palmitate treatment of rat islets increased ER chaperone levels along with PERK/IRE1 and the ER degradation machinery $[17,50]$. In agreement with these studies, we found that 10-week-old BTBR male mice showed increased expression of ER chaperones including Bip, EDEM and DNAJB9 $[3,4,51]$.

In a model for $\beta$-cell failure in obesity-associated type 2 diabetes proposed by Marc Prentki and Christopher Nolan [52], ER stress has been articulated as a cause for early $\beta$-cell failure. Thus, in the BTBR model where nearly half of the $\beta$ cells are decompensated [38], we propose that increased ER stress augments $\beta$-cell apoptosis.

3.1. Novel Observations/Hypotheses from the Connexios Network Biology Platform Linking ER Stress to Beta Cell Dysfunction in the Obese Diabetic BTBR Mouse Model.

(1) We found a significant reduction in the pancreas oxidative protein folding machinery, PDIA2 and ERO1B [53], which are known to introduce disulfide bonds in the insulin protein. As such, we expect functional insulin levels to be decreased leading to severe hyperglycemia in the BTBR model. From a $\beta$-cell perspective, this may represent an adaptive mechanism that reduces ER stress and subsequent $\beta$-cell apoptosis induced by an insulin overload. In keeping with this, partial loss of ERO1b function is known to reduce ER stress in MIN6 cells [53]. 


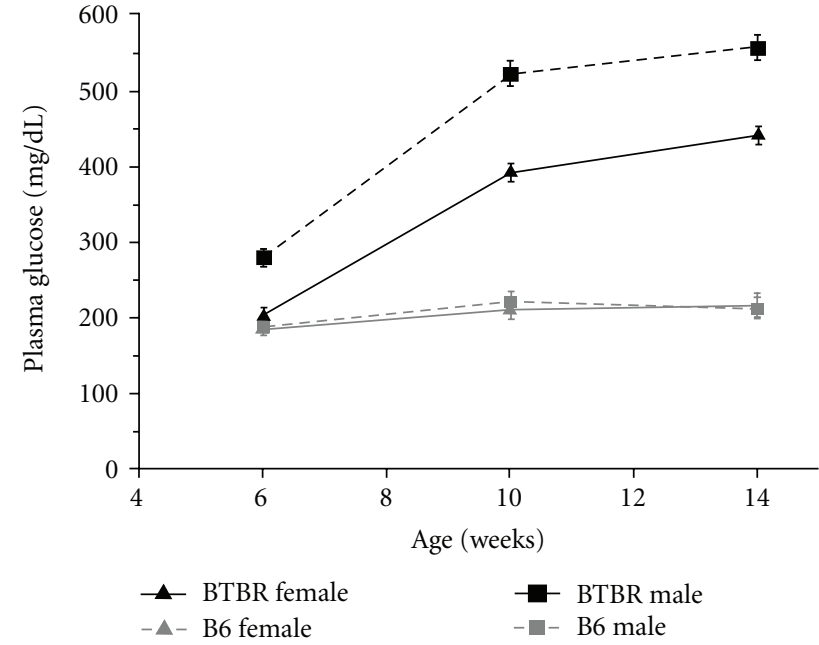

(a)

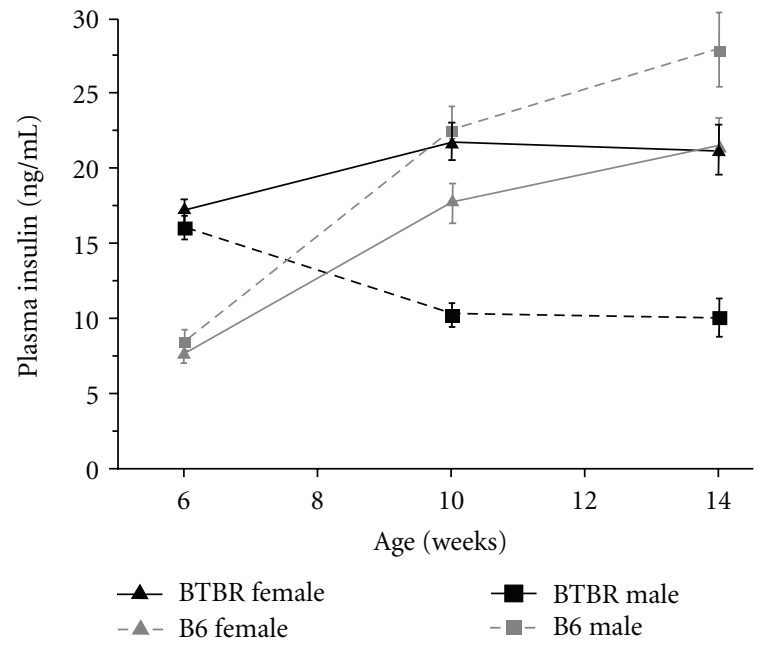

(b)

Figure 3: Plasma glucose and insulin levels following a $4 \mathrm{~h}$ fast in ob/ob mice as a function of age. BTBR are shown in black filled symbols and B6 in gray open symbols. Males are shown as dashed lines and females as solid lines (Reproduced from [38]).

(2) We observed a loss of an ATF4/ATF6-mediated compensatory mechanism that regulates aminoacid homeostasis/transport and phosphatidylcholine biosynthesis. This mechanism is critical to regulate the antioxidant system and ER membrane biogenesis [32]. In this regard, it will be interesting to verify the transcription status of ATF-dependent genes that function in amino acid transport/homeostasis and phosphatidylcholine biosynthesis in $\beta$ cells. This will have implications for the pathogenesis seen in type 2 diabetes.

(3) We detected a significant increase in expression of genes involved in dolichol-phosphate-mannose synthesis and transfer reactions. This may be an adaptive mechanism to improve trimming and proper folding of $\mathrm{N}$-glycans in obese diabetic $\beta$ cells. Understanding the regulation of the above genes in UPR will be an interesting area of research for maintaining $\beta$ cell health in type 2 diabetes.

(4) Increased expression of MAP3K5 (ASK1) may indicate an IRE1-ASK1-mediated induction of apoptotic signals in response to chronic ER stress. Importance of ASK1 in ER stress-mediated cell death has been demonstrated in neuronal cells [36]. Hence, it would be of interest to ascertain whether a similar mechanism is involved in a chronic glucolipotoxic and inflammatory conditions in obese diabetic pancreatic beta cells.

3.2. Summary of Connexios Network Biology Platform Contribution to the Understanding of ER Stress-Mediated Pathogenesis in Obese Diabetic BTBR Mice. Using the Connexios ER stress module, we predict that ER stress contributes significantly to beta cell apoptosis in the obese type 2 diabetic model, BTBR. This is the first report to address multiple arms of ER stress in the BTBR pancreatic islet $\beta$ cells. Our findings suggest that increased/sustained ER stress in pancreatic $\beta$ cells under obese type 2 diabetic conditions leads to:

(1) inhibition of protein synthesis and increased expression of ER folding/degradation machinery to clear misfolded protein accumulation;

(2) decreased insulin production due reduced protein folding, a protective mechanism to maintain $\beta$-cell health;

(3) increased dolichol-phosphate-mannose synthesis and transfer reactions to regulate misfolded protein accumulation;

(4) dysregulation of adaptive mechanisms including aminoacid metabolism and phosphatidylcholine synthesis that regulate translation, protein synthesis, antioxidants, and ER membrane biogenesis, respectively;

(5) reduced insulin gene transcription;

(6) increased stress signaling-mediated $\beta$-cell apoptosis.

In conclusion, we provide an in silico ER stress network module that can be used to study existing gene expression data to generate novel, testable hypotheses, and design functional studies in several biological systems. Such an integrative network approach holds potential to address multiple disease phenotypes and pharmacological treatments through in silico models of cellular organelles consolidated across multiple functions and cell types.

\section{Acknowledgment}

The authors thank all the past and present members of the Systems Biology group in Connexios Life Sciences Pvt. Ltd. for their contributions towards curation of the ER signaling pathways included in the paper. 


\section{References}

[1] O. Baumann and B. Walz, "Endoplasmic reticulum of animal cells and its organization into structural and functional domains," International Review of Cytology, vol. 205, pp. 149214, 2001.

[2] D. Ron and P. Walter, "Signal integration in the endoplasmic reticulum unfolded protein response," Nature Reviews Molecular Cell Biology, vol. 8, no. 7, pp. 519-529, 2007.

[3] I. Köllner, B. Sodeik, S. Schreek et al., "Mutations in neutrophil elastase causing congenital neutropenia lead to cytoplasmic protein accumulation and induction of the unfolded protein response," Blood, vol. 108, no. 2, pp. 493-500, 2006.

[4] N. Dejeans, N. Tajeddine, R. Beck et al., "Endoplasmic reticulum calcium release potentiates the ER stress and cell death caused by an oxidative stress in MCF-7 cells," Biochemical Pharmacology, vol. 79, no. 9, pp. 1221-1230, 2010.

[5] H. Mujcic, T. Rzymski, K. M. Rouschop et al., "Hypoxic activation of the unfolded protein response (UPR) induces expression of the metastasis-associated gene LAMP3," Radiotherapy and Oncology, vol. 92, no. 3, pp. 450-459, 2009.

[6] R. Chapman, C. Sidrauski, and P. Walter, "Intracellular signaling from the endoplasmic reticulum to the nucleus," Annual Review of Cell and Developmental Biology, vol. 14, pp. 459-485, 1998.

[7] D. J. DeGracia and H. L. Montie, "Cerebral ischemia and the unfolded protein response," Journal of Neurochemistry, vol. 91, no. 1, pp. 1-8, 2004.

[8] G. R. Medigeshi, A. M. Lancaster, A. J. Hirsch et al., "West nile virus infection activates the unfolded protein response, leading to CHOP induction and apoptosis," Journal of Virology, vol. 81, no. 20, pp. 10849-10860, 2007.

[9] G. G. Lavery, E. A. Walker, N. Turan et al., "Deletion of hexose6-phosphate dehydrogenase activates the unfolded protein response pathway and induces skeletal myopathy," Journal of Biological Chemistry, vol. 283, no. 13, pp. 8453-8461, 2008.

[10] Q. Li, Z. Liu, J. Guo et al., "Cholesterol overloading leads to hepatic L02 cell damage through activation of the unfolded protein response," International Journal of Molecular Medicine, vol. 24, no. 4, pp. 459-464, 2009.

[11] H. R. Park, A. Tomida, S. Sato et al., "Effect on tumor cells of blocking survival response to glucose deprivation," Journal of the National Cancer Institute, vol. 96, no. 17, pp. 1300-1310, 2004.

[12] K. L. Lipson, S. G. Fonseca, S. Ishigaki et al., "Regulation of insulin biosynthesis in pancreatic beta cells by an endoplasmic reticulum-resident protein kinase IRE1," Cell Metabolism, vol. 4, no. 3, pp. 245-254, 2006.

[13] R. J. Kaufman, S. H. Back, B. Song, J. Han, and J. Hassler, "The unfolded protein response is required to maintain the integrity of the endoplasmic reticulum, prevent oxidative stress and preserve differentiation in $\beta$-cells," Diabetes, Obesity and Metabolism, vol. 12, no. 2, pp. 99-107, 2010.

[14] S. Oyadomari, K. Takeda, M. Takiguchi et al., "Nitric oxideinduced apoptosis in pancreatic $\beta$ cells is mediated by the endoplasmic reticulum stress pathway," Proceedings of the National Academy of Sciences of the United States of America, vol. 98, no. 19, pp. 10845-10850, 2001.

[15] S. Oyadomari, A. Koizumi, K. Takeda et al., "Targeted disruption of the chop gene delays endoplasmic reticulum stress-mediated diabetes," Journal of Clinical Investigation, vol. 109, no. 4, pp. 525-532, 2002.

[16] B. Qian, H. Wang, X. Men et al., "TRIB3 is implicated in glucotoxicity-and endoplasmic reticulum-stress-induced beta-cell apoptosis," Journal of Endocrinology, vol. 199, no. 3, pp. 407-416, 2008.

[17] P. Marchetti, M. Bugliani, R. Lupi et al., "The endoplasmic reticulum in pancreatic beta cells of type 2 diabetes patients," Diabetologia, vol. 50, no. 12, pp. 2486-2494, 2007.

[18] S. J. Marciniak, C. Y. Yun, S. Oyadomari et al., "CHOP induces death by promoting protein synthesis and oxidation in the stressed endoplasmic reticulum," Genes \& Development, vol. 18, no. 24, pp. 3066-3077, 2004.

[19] M. C. Åkerfeldt, J. Howes, J. Y. Chan et al., "Cytokine-induced $\beta$-cell death is independent of endoplasmic reticulum stress signaling," Diabetes, vol. 57, no. 11, pp. 3034-3044, 2008.

[20] S. Del Prato, P. Marchetti, and R. C. Bonadonna, "Phasic insulin release and metabolic regulation in type 2 diabetes," Diabetes, vol. 51, supplement 1, pp. S109-S116, 2002.

[21] S. Del Prato and P. Marchetti, "Beta-and alpha-cell dysfunction in type 2 diabetes," Hormone and Metabolic Research, vol. 36, no. 11-12, pp. 775-781, 2004.

[22] D. T. Rutkowski and R. J. Kaufman, "A trip to the ER: coping with stress," Trends in Cell Biology, vol. 14, no. 1, pp. 20-28, 2004.

[23] R. J. Kaufman, "Stress signaling from the lumen of the endoplasmic reticulum: coordination of gene transcriptional and translational controls," Genes \& Development, vol. 13, no. 10, pp. 1211-1233, 1999.

[24] H. P. Harding, Y. Zhang, and D. Ron, "Protein translation and folding are coupled by an endoplasmic-reticulum-resident kinase," Nature, vol. 397, no. 6716, pp. 271-274, 1999.

[25] J. Hollien, J. H. Lin, H. Li, N. Stevens, P. Walter, and J. S. Weissman, "Regulated Ire1-dependent decay of messenger RNAs in mammalian cells," Journal of Cell Biology, vol. 186, no. 3, pp. 323-331, 2009.

[26] S. W. Kang, N. S. Rane, S. J. Kim, J. L. Garrison, J. Taunton, and R. S. Hegde, "Substrate-specific translocational attenuation during ER stress defines a pre-emptive quality control pathway," Cell, vol. 127, no. 5, pp. 999-1013, 2006.

[27] H. P. Harding, Y. Zhang, H. Zeng et al., "An integrated stress response regulates amino acid metabolism and resistance to oxidative stress," Molecular Cell, vol. 11, no. 3, pp. 619-633, 2003.

[28] S. B. Cullinan and J. A. Diehl, "Coordination of ER and oxidative stress signaling: the PERK/Nrf2 signaling pathway," International Journal of Biochemistry \& Cell Biology, vol. 38, no. 3, pp. 317-332, 2006.

[29] S. B. Cullinan, D. Zhang, M. Hannink, E. Arvisais, R. J. Kaufman, and J. A. Diehl, "Nrf2 is a direct PERK substrate and effector of PERK-dependent cell survival," Molecular and Cellular Biology, vol. 23, no. 20, pp. 7198-7209, 2003.

[30] S. B. Cullinan and J. A. Diehl, "PERK-dependent activation of Nrf2 contributes to redox homeostasis and cell survival following endoplasmic reticulum stress," Journal of Biological Chemistry, vol. 279, no. 19, pp. 20108-20117, 2004.

[31] H. Bommiasamy, S. H. Back, P. Fagone et al., "ATF6 $\alpha$ induces XBP1-independent expansion of the endoplasmic reticulum," Journal of Cell Science, vol. 122, no. 10, pp. 1626-1636, 2009.

[32] R. Sriburi, S. Jackowski, K. Mori, and J. W. Brewer, "XBP1: a link between the unfolded protein response, lipid biosynthesis, and biogenesis of the endoplasmic reticulum," Journal of Cell Biology, vol. 167, no. 1, pp. 35-41, 2004.

[33] D. Ron and R. Y. Hampton, "Membrane biogenesis and the unfolded protein response," Journal of Cell Biology, vol. 167, no. 1, pp. 23-25, 2004. 
[34] E. Szegezdi, S. E. Logue, A. M. Gorman, and A. Samali, "Mediators of endoplasmic reticulum stress-induced apoptosis," EMBO Reports, vol. 7, no. 9, pp. 880-885, 2006.

[35] M. Matsumoto, M. Minami, K. Takeda, Y. Sakao, and S. Akira, "Ectopic expression of CHOP (GADD153) induces apoptosis in M1 myeloblastic leukemia cells," FEBS Letters, vol. 395, no. 2-3, pp. 143-147, 1996.

[36] H. Nishitoh, A. Matsuzawa, K. Tobiume et al., "ASK1 is essential for endoplasmic reticulum stress-induced neuronal cell death triggered by expanded polyglutamine repeats," Genes \& Development, vol. 16, no. 11, pp. 1345-1355, 2002.

[37] X. Z. Wang and D. Ron, "Stress-induced phosphorylation and activation of the transcription factor CHOP (GADD153) by p38 MAP kinase," Science, vol. 272, no. 5266, pp. 1347-1349, 1996.

[38] S. M. Clee, S. T. Nadler, and A. D. Attie, "Genetic and genomic studies of the BTBR ob/ob mouse model of type 2 diabetes," American Journal of Therapeutics, vol. 12, no. 6, pp. 491-498, 2005.

[39] M. P. Keller, Y. Choi, P. Wang et al., "A gene expression network model of type 2 diabetes links cell cycle regulation in islets with diabetes susceptibility," Genome Research, vol. 18, no. 5, pp. 706-716, 2008.

[40] T. Ranheim, C. Dumke, K. L. Schueler, G. D. Cartee, and A. D. Attie, "Interaction between BTBR and C57BL/6J genomes produces an insulin resistance syndrome in (BTBR $\mathrm{x}$ C57BL/6J) F1 mice," Arteriosclerosis, Thrombosis, and Vascular Biology, vol. 17, no. 11, pp. 3286-3293, 1997.

[41] J. P. Stoehr, S. T. Nadler, K. L. Schueler et al., "Genetic obesity unmasks nonlinear interactions between murine type 2 diabetes susceptibility loci," Diabetes, vol. 49, no. 11, pp. 1946-1954, 2000.

[42] G. C. Weir and S. Bonner-Weir, "Five of stages of evolving $\beta$ cell dysfunction during progression to diabetes," Diabetes, vol. 53, supplement 3, pp. S16-S21, 2004.

[43] J. Averous, A. Bruhat, C. Jousse, V. Carraro, G. Thiel, and P. Fafournoux, "Induction of CHOP expression by amino acid limitation requires both ATF4 expression and ATF2 phosphorylation," Journal of Biological Chemistry, vol. 279, no. 7, pp. 5288-5297, 2004.

[44] S. Gottesman, S. Wickner, and M. R. Maurizi, "Protein quality control: triage by chaperones and proteases," Genes \& Development, vol. 11, no. 7, pp. 815-823, 1997.

[45] Y. Shen and L. M. Hendershot, "ERdj3, a stress-inducible endoplasmic reticulum dnaJ homologue, serves as a cofactor for BiP's interactions with unfolded substrates," Molecular Biology of the Cell, vol. 16, no. 1, pp. 40-50, 2005.

[46] S. F. Göthel and M. A. Marahiel, "Peptidyl-prolyl cis-trams isomerases, a superfamily of ubiquitous folding catalysts," Cellular and Molecular Life Sciences, vol. 55, no. 3, pp. 423436, 1999.

[47] G. Kozlov, P. Määttänen, D. Y. Thomas, and K. Gehring, "A structural overview of the PDI family of proteins," FEBS Journal, vol. 277, no. 19, pp. 3924-3936, 2010.

[48] B. Knoblach, B. O. Keller, J. Groenendyk et al., "ERp19 and ERp46, new members of the thioredoxin family of endoplasmic reticulum proteins," Molecular \& Cellular Proteomics, vol. 2, no. 10, pp. 1104-1119, 2003.

[49] R. Sriburi, S. Jackowski, K. Mori, and J. W. Brewer, "XBP1: a link between the unfolded protein response, lipid biosynthesis, and biogenesis of the endoplasmic reticulum," Journal of Cell Biology, vol. 167, no. 1, pp. 35-41, 2004.
[50] E. Karaskov, C. Scott, L. Zhang, T. Teodoro, M. Ravazzola, and A. Volchuk, "Chronic palmitate but not oleate exposure induces endoplasmic reticulum stress, which may contribute to INS-1 pancreatic $\beta$-cell apoptosis," Endocrinology, vol. 147, no. 7, pp. 3398-3407, 2006.

[51] A. Finka, R. U. Mattoo, and P. Goloubinoff, "Meta-analysis of heat-and chemically upregulated chaperone genes in plant and human cells," Cell Stress and Chaperones, vol. 16, no. 1, pp. 1531, 2011.

[52] M. Prentki and C. J. Nolan, "Islet $\beta$ cell failure in type 2 diabetes," Journal of Clinical Investigation, vol. 116, no. 7, pp. 1802-1812, 2006.

[53] E. Zito, K. T. Chin, J. Blais, H. P. Harding, and D. Ron, "ERO1$\beta$, a pancreas-specific disulfide oxidase, promotes insulin biogenesis and glucose homeostasis," Journal of Cell Biology, vol. 188, no. 6, pp. 821-832, 2010. 

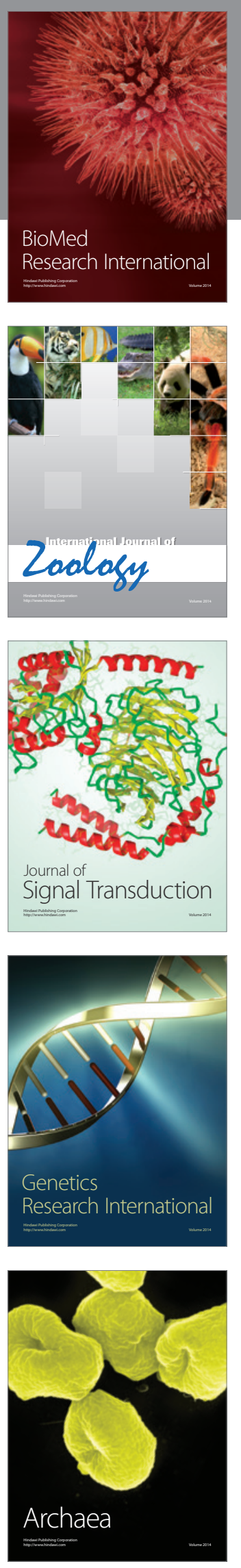
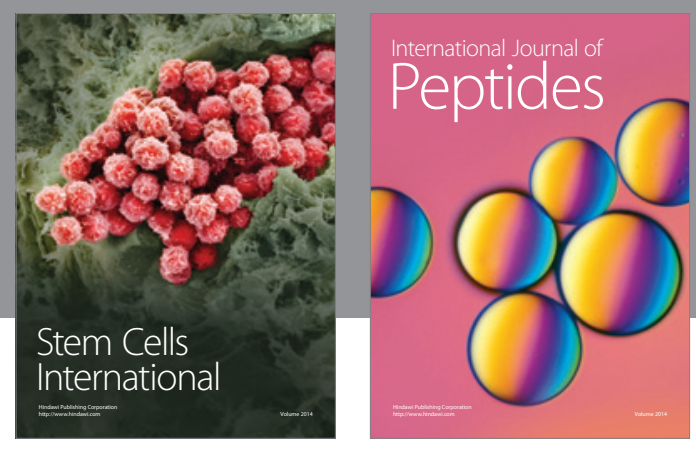

Submit your manuscripts at

http://www.hindawi.com
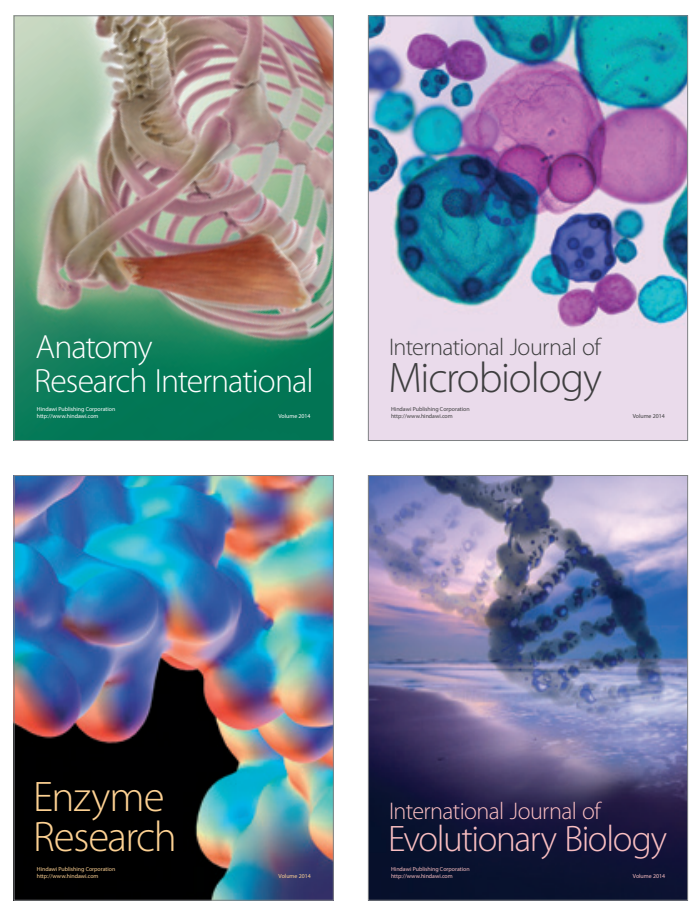
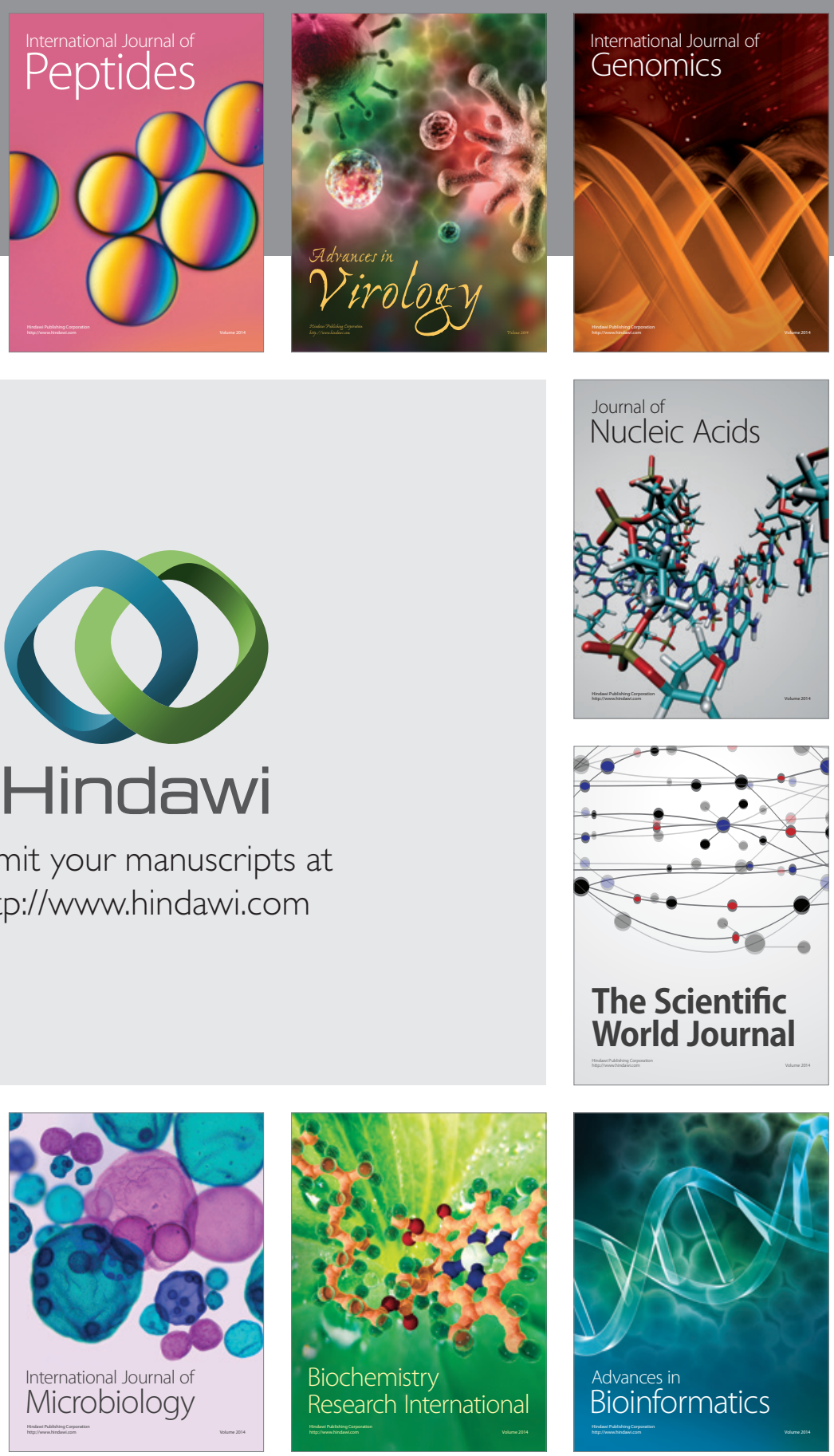

The Scientific World Journal
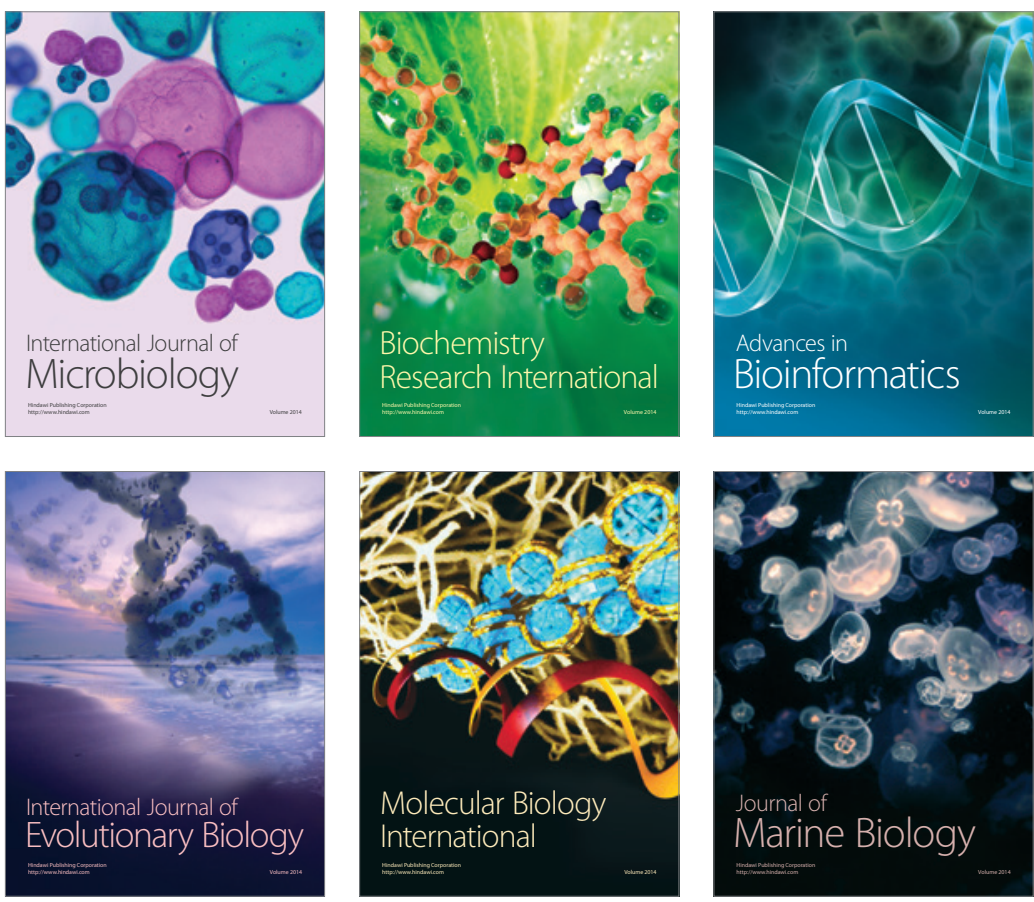